\title{
Automated Video-Assisted Smoking Treatment
}

National Cancer Institute

\section{Source}

National Cancer Institute. Automated Video-Assisted Smoking Treatment. NCI

Thesaurus. Code C161003.

A fully automated and interactive smartphone-based treatment program that comprises interactive text messaging, images and audio/video clips to enable smoking cessation treatment content to be presented in an interactive, structured format anytime and anywhere. 